\title{
A Method of Deriving Subgroups of a Population: A Study of Craniofacial Taxonomy
}

\author{
W. J. HIRSCHFELD, ${ }^{1}$ R. E. MOYERS AND D. H. ENLOW \\ Center for Human Growth and Development, The University of Michigan, \\ Ann Arbor, Michigan 48104
}

\author{
KEY WORDS Numerical taxonomy - Cluster analysis \\ Craniofacial measurements.
}

\begin{abstract}
Subgroups within a population are often difficult to discover and describe except by subjective methods. In this study, cluster analysis (numerical taxonomy) methods were used on selected craniofacial measurements obtained from 308 North American White children of both sexes in the age range 6-18 to derive categories of skeletal facial types.

Two different cluster analysis approaches were used in conjunction with a separate overall evaluation of facial balance, an independent measure of maxillo-mandibular relationship ( $A B / F O P$ ), and a traditional classification (Angle). The categories derived rest on corroborative and overlapping evidence from each of those methods.

The categories were examined to determine if it is possible to classify a sample by means of cluster analysis, the size and discreteness of each class, how they compare with the Angle classification of the same sample, and the percentage of individuals that may be identified unequivocally by this classifcation scheme.

Five categories were obtained. Labeled Category A - Category E, they show some correspondence to the Angle Classes I, II, and III, but categories A, B, and $\mathrm{C}$ appear to be subgroups, heretofore undetected, of Angle Class II. Categories D and E correspond to Angle Classes I and III, respectively. The categories are more realistic and informative than the Angle classes. Each category is reported along with its distinguishing skeletal characteristics.
\end{abstract}

A common problem in anthropology is the taxonomic one of defining and labeling subgroups of a population. Traditionally, this process has been carried out by methods that are essentially subjective and, therefore, perhaps unable to deal with the whole of a set of variables in an efficient, quantifiable, and reproducible manner.

Multivariate methods have been developed and applied in taxonomy in an effort to improve the evaluation of an individual as a whole, and to make classification schemes more objective. By means of numerical operations on the data, these methods derive subgroups for further study, thus serving as heuristic tools for the investigator (Blackith and Reyment, '71). This is a report of the application of methods of exploratory data analysis to the general problem of discovering subgroups of a population.

We wished to know what skeletal types may be found in a given population, and to be able to find them in an objective way. That is, we wanted groups to emerge from within the data, as opposed to being imposed upon the data by us. Accordingly, we chose the multivariate taxonomic approach specifically designed for this purpose, viz. the methods of numerical taxonomy known in the statistical literature as cluster analysis.

Other multivariate techniques have been used by Brown, Barrett and Darroch ('65) and by Howells ('69, '71) in craniofacial comparisons of ethnic groups. The present work is concerned with determining sub-

1 Present address: Chicago State University, 95 th and King Drive, Chicago, Illinois 60628. 
groups rather than with comparisons of existing groups. We must emphasize that the methods reported in this paper are equally applicable to the derivation of groupings in other sorts of anthropologic data (e.g., blood groups, body stature, electrophoretic patterns).

As pointed out by Gower ('67) there are many techniques of cluster analysis, and it is difficult to judge their relative merits, because a cluster is not a well defined concept. The basic criterion for a cluster is that the mathematical distance between its center and any point in the cluster must be less than the distance between its center and the center of any other cluster. In other words, a point is assigned to that cluster in which the pointcenter distance is a minimum. The ill-defined concept of a cluster is caused by the facts that this criterion may be expressed and implemented in a variety of ways, and that a cluster may have a spherical, ellipsoidal, or other shape.

Besides being used for the clustering of objects, the same techniques may be used in the analysis of the variables in a multivariate set. In much the same way as factor analysis, cluster analysis may uncover in a set of variables the basic subset that provides most of the information. Both kinds of applications have been used in the present studies.

Most of the techniques of numerical taxonomy currently in use have been reviewed by Rohlf ('70) who also provides the important reminder that these methods are not statistical in nature (for elaboration, see the work cited).
The purpose of the work is to answer the following questions: (1) is it possible to classify a sample of a given population into different skeletal facial types by the methods of cluster analysis; (2) if so, what are the sizes, characteristics, number and discreteness of the classes; (3) how do the classes compare to traditional subjective methods of classification (Angle, '07); (4) what percentage of individuals examined may be identified unequivocally with respect to the classes derived?

\section{METHODS}

The data base

All the data used in these studies were obtained from lateral cephalograms of 308 North American White children in the age range 6-18, unstratified with respect to age or sex groupings. This material had been evaluated subjectively with respect to presence and type of malocclusion (Angle classification) for other purposes and it had been determined that all three Angle classes were represented. Therefore, the same material afforded us an ideal opportunity to test the objective taxonomic approach against the subjective.

The data are of the following kinds:

(1) 13 variables (see table 1) pertaining to structural counterparts. These are the same data used by Enlow in his recent anatomic studies. The method of measurement, reported elsewhere (Enlow, Kuroda and Lewis, '71a), results in an integer value in the scale $1-7$. A value of 4 means that the structural equivalents are in balance. Values of 5,6 , or 7 indicate imbalance greater than 1,2 , or 3 standard

TABLE 1

List of structural counterparts variables (Enlow and Moyers, '71; Enlow et al., '71a,b)

\begin{tabular}{|c|c|}
\hline Var. no. & Description \\
\hline 1 & Overall balance measured from $A$ and $B$ points \\
\hline 2 & Overall balance measured from SPr and IPr \\
\hline 3 & Alignment of posterior cranial base \\
\hline 4 & Alignment of ramus of mandible \\
\hline 5 & Posterior cranial base-ramus balance measured skeletally \\
\hline 6 & Posterior cranial base-ramus balance measured dentally \\
\hline 7 & $\begin{array}{l}\text { Maxillary arch-corpus of mandible balance measured skeletally from } \\
A \text { and } B \text { points }\end{array}$ \\
\hline 8 & Maxillary arch-corpus balance measured dentally from $A$ and $B$ \\
\hline 9 & Maxillary arch-corpus balance measured skeletally from SPr and IPr \\
\hline 10 & Maxillary arch-corpus balance measured dentally from SPr and IPr \\
\hline 11 & Vertical midface height \\
\hline 12 & Alignment of corpus of mandible \\
\hline 13 & Deviation of plane of occlusion of corpus away from reference line \\
\hline
\end{tabular}


deviations in one direction, values of 3,2 , or 1 similar imbalance in the opposite direction. The anatomical significance of the direction of imbalance varies with the particular variable, as described in the work cited.

(2) An overall evaluation of each profile done by D. H. Enlow on the basis of the first two variables listed in table 1. Maxillary protrusion is associated with values of 5-7 for both these variables, and is designated herein as MAX. Mandibular protrusion, designated MAN, is associated with values of $1-3$. BAL goes with a balanced profile and a balanced number of values pivoting around 4 .

(As the emphasis of this paper is taxonomy, not morphology, a detailed description of the method of obtaining these measurements is inappropriate. The reader may rest assured that they are valid for the present purposes, and may consult the works cited for their significance in craniofacial biology and orthodontics.)

(3) An evaluation done by one person with regard to the Angle classification of occlusion as stated above.

(4) The measurement of direction and magnitude of deviation from the mean of the angle of intersection of the line A pt.$B$ pt. with the functional occlusal plane (AB/FOP). This angle has been found to remain relatively constant with time (Hirschfeld and Moyers, manuscript in preparation) and provides an effective measure of the maxillo-mandibular relationship existing in a given subject. The mean vaule used is $88.6^{\circ}$ with a standard deviation of $3.2^{\circ}$. Data were recorded as $\pm 1,2$, or 3 standard deviations, " +" signifying mandibular protrusion and "-" signifying maxillary protrusion.

(5) A discriminator variable formed by summating the values of variables 3-13 in table 1. This was done for each subject primarily for the purpose of seeking clusters in Prim networks as will be explained later. Note that the variables used to form this discriminator are different from those used in forming the MAX, BAL, MAN categories. Because each variable has the numerical range $1-7$, the theoretical minimum for the discriminator is $11 \times 1=11$, the maximum is $11 \times 7=77$.

(6) Relative location in a Prim network (see below). As this information can be gained only by inspection of the network, it is of little value by itself. However, when combined with other data it identifies the subjects that form any groupings found in the networks.

(7) The DICO cluster (see below) in which a subject is located. Because DICO clusters are each formed around a different single subject (the center), the location of any other subject tells which center that individual most resembles.

\section{Cluster analysis and numerical taxonomy}

Tryon's VCLUST (cluster analysis of variables). Part of a system of computer programs developed by Tryon and his coworkers, VCLUST is designed specifically to gather together, out of a full set of variables, subsets of variables that are collinear (i.e., definitely positively correlated). Each variable within any one subset is more similar in behavior to any other variable in that same subset, than it is to any variable in any other subset. Together with each such subset is computed a reliability coefficient, which gives a measure of confidence that the user may have in the degree of similarity of the variables. In the present studies, a minimum of 0.7 was set for this coefficient. Values below this level were not considered sufficient evidence for similarity of the variables involved.

An important consequence of collinearity is that since all the variables in a collinear group are similar, one representative variable may be taken from each group, and the entire set of variables reduced to a smaller number representative of the behavior of the full set.

A full account of the methods of $V$ analysis (variable analysis) is given by Tryon and Bailey ('71).

Prim nearest-neighbor network. This is a method of numerical taxonomy used for the analysis of objects, rather than of variables. The Prim network consists of symbols representing the objects under study (the individuals of the sample) connected by lines, the lengths of which are proportional to the mathematical distances between the points. Each object is connected to the object closest to it (i.e., to its nearest neighbor). The choice of the first point in the network (the first indi- 
vidual) is arbitrary. Thus, a multivariate comparison can be made among individuals, and some graphic representation constructed of the interrelationships among all the individuals in a sample.

For more information consult the articles by Farris ('70) and by Prim ('57).

The dissimilarity coefficient (Sokal and Sneath, '63). This is a distance measure suitable for use with qualitative data. For each pair of subjects, a count is made of all corresponding variables for which the values are not the same. This number is then divided by the total number of variables to yield the dissimilarity coefficient (DICO). Thus, if, for a given pair of subjects, all the variables have the same value, the number of dissimilar variables will be zero, and the DICO will then also be zero. If all the variables are dissimilar, the DICO will be unity. The DICO, therefore, is a number in the range $0-1$. This approach is most often used when dealing with dichotomous data, for example, plus vs. minus, present vs. absent, or zero vs. one. In the present application the data are integers from 1-7, each variable having a value somewhere in that range.

\section{The application of cluster analysis to the data base}

Following preliminary studies, a Prim network based on variables 3-13 was selected as giving the best spread of subjects. On this network, the value of the discriminator variable was entered alongside each subject's identification number. By trial and error, the ranges of values of the discriminator were determined to minimize overlap among groupings of subjects. These ranges were 13-30, designated as gamma; 31-48, beta; 49 and above, alpha.

A separate set of clusters was formed by the DICO method. Each subject then was a member of one DICO and one Prim grouping. The crucial question was - are the characteristics of the two sets of groups the same?

If they are, then the same classification was obtained by two different methods of numerical taxonomy, thus greatly increasing the faith one may have that the classifications are correct.

The final phase of the work, and the test of the question, was the listing of all subjects so that all the data (Angle Class,
AB/FOP angle, etc.) could be compared. The lists were ordered arbitrarily in terms of the DICO clusters.

\section{Cluster analysis of variables}

This consisted of the application of the VCLUST technique described earlier to determine if the information conveyed by the full set of 13 variables could be approximated to an acceptable degree by a smaller number of variables.

\section{RESULTS}

\section{Numerical taxonomy}

Figure 1 shows, with symbols in place of identification numbers, the Prim network obtained using variables 3-13 for each of the 308 subjects. Once the groupings were established, the anatomic characteristics of each subject in each group were ascertained from the original data. Definite differences appeared among the groups as follows.

The group of subjects symbolized by hexagons was characterized by net maxillary protrusion, the cause of the imbalance being either a relatively long maxilla or a relatively short mandible. This group may be considered to be Angle Class II subjects of varying degree of protrusion. At its periphery, it blends with the next group, the triangles.

The group of subjects marked by triangles may be considered to be Angle Class I, exhibiting balanced profiles. Again, there exists a spectrum going from profiles bordering on maxillary protrusion to those bordering on mandibular protrusion. Note, however, that the degree of overlap between "triangle" and "hexagon" is greater than that between "triangle" and "circle."

The last group, the circles, represents Angle Class III, or net mandibular protrusion. In this group, two subgroups emerged that showed a difference in the nature of the imbalance. That is, inspection of the original data on the subjects showed the subgroup marked by an $\mathrm{X}$ within the circle to have values of 1 or 2 for most variables as opposed to values of 2 or 3 for the same variables in the other subgroup (see numerical scale, page 280). The remainder of the circle group blends somewhat with the triangle, or balanced profile, group in 


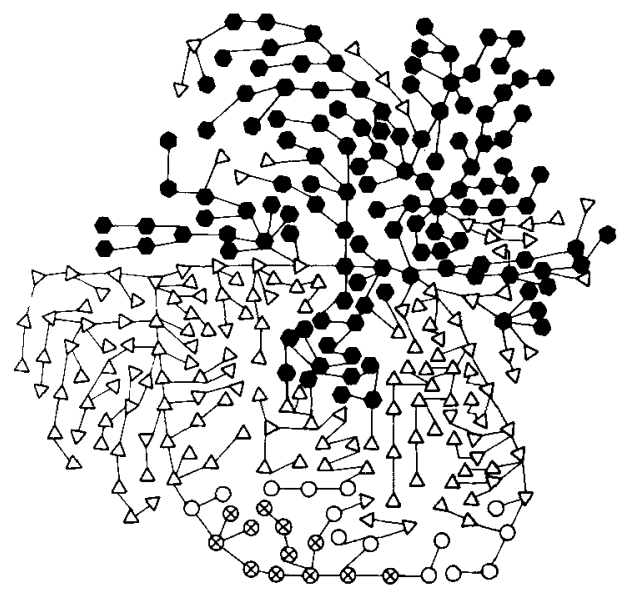

- Primorily Angle Class II net moxillary protrusion

$\triangle$ Primarily Angle Class I; balanced maxillo-mandibular relationship

O All Primarily Angle Class $\mathrm{II}$; nef mandibulor protrusion

- Angle Class III subgroup

\author{
Value of Discriminator Voriable $\sum_{i=1}^{11} v_{i}$ \\ - 49 and above \\ $\triangle 31-48$ \\ 25-30 \\ 824 and below
}

Fig. 1 Prim network in symbolic form. Geometric figures have been substituted for the subjects' identification numbers in accordance with the value of the discriminator variable. The discriminator variable and the network are based on variables $3-13$ in the list given in table 1 .

accordance with the degree of severity of mandibular protrusion.

Hereafter, the hexagon group will be referred to as alpha, the triangle as beta and the circle as gamma to conform with the terminology used with respect to the discriminator variables. Because there are hints of subgroups of gamma, the one with the higher values of the discriminator variable will be called gamma-1, and the one with the lower values will be gamma-2.

Of the 308 subjects, $126(40.9 \%)$ fell in the alpha group, $156(50.7 \%)$ in the beta, and $26(8.4 \%)$ in the gamma. Of the latter, $14(4.5 \%$ of the total sample) were in gamma-1, and $12(3.9 \%$ of the total sample) were in gamma-2.

Turning now to DICO clustering, five different clusters of subjects emerged. Unambiguous membership in clusters occurred for $243(78.9 \%)$ of the subjects. The other $65(21.1 \%)$ subjects were equally similar to two or more of the clusters and, therefore, could not be classified unambiguously. These subjects are to the DICO clusters as the borderline subjects are to the Prim clusters, and will be analyzed separately after the presentation of the results obtained for the unambiguously classifiable subjects.

Table 2 gives the size of the DIFCO clusters together with those of the Prim clusters. The percentage distributions of subjects in the two kinds of clusters gave some clue as to possible correspondence between the two methods. The largest Prim cluster was beta, the largest DICO cluster was 4 . Could these contain the same subjects?

A more detailed comparison was made as follows. For each single cluster, the mean value of each variable was compared with the grand mean for that variable taken over the whole sample. The information was recorded as $\mathrm{A}$ for above the grand mean, $B$ for below the grand mean and is shown in table 3. (A dash indicates a value at or close to the grand mean.) In this way, the patterns of deviations of the variables relative to grand means could be compared for Prim and DICO clusters. The most striking resemblance is between Prim gamma and DICO 5. This was verified by direct comparison of the subject identification numbers; gamma and 5 are essentially alike. In the same way, beta and 4 are similar, and alpha corresponds to all of 1,2 , and 3 . The latter suggests that alpha, the Angle Class II type, is composed of three subgroups not revealed by the Prim network.

This greater sensitivity in revealing subgroups dictated the choice of the DICO clusters over the Prim for drawing together all the information gathered for each subject. This will be done here by 
TABLE 2

Relative sizes of Prim and DICO clusters

\begin{tabular}{lccc}
\hline Group & $\begin{array}{c}\text { No. in } \\
\text { size group }\end{array}$ & $\begin{array}{c}\text { Per cent of } \\
\text { Per cent of } \\
\text { total sample }\end{array}$ & $\begin{array}{c}\text { onambiguously } \\
\text { classifiable } \\
\text { subjects }\end{array}$ \\
\hline Prim alpha & 126 & 40.9 & - \\
Prim beta & 156 & 50.7 & - \\
Prim gamma & 26 & 8.4 & - \\
DICO 1 & 32 & 10.4 & 13.2 \\
DICO 2 & 29 & 9.4 & 11.9 \\
DICO 4 & 35 & 11.4 & 14.4 \\
DICO 5 & 99 & 32.1 & 40.7 \\
\hline
\end{tabular}

TABLE 3

\begin{tabular}{|c|c|c|c|c|c|c|c|c|c|c|c|}
\hline \multirow[b]{2}{*}{ Group } & \multicolumn{11}{|c|}{ Variable } \\
\hline & 3 & 4 & 5 & 6 & 7 & 8 & 9 & 10 & 11 & 12 & 13 \\
\hline Prim alpha & - & A : & - & A & $\mathrm{A}_{\mathrm{B}} 2$ & $\mathrm{~A}_{3}$ & $\mathrm{~A}$ & $\underline{A}$ & $\underline{A}$ & $\mathrm{~A}$ & A \\
\hline Prim gamma & $\overline{\mathrm{B}}$ & $\overline{\mathrm{B}}$ & - & $\overline{\mathrm{B}}$ & $\mathrm{B}$ & $\bar{B}$ & $\mathrm{~B}$ & $\bar{B}$ & $\bar{B}$ & $\bar{B}$ & B \\
\hline DICO 1 & - & A & A & A & A & A & A & A & A & 一 & - \\
\hline DICO 2 & A & A & $B$ & - & $\mathrm{A}$ & A & A & A & - & A & - \\
\hline DICO 3 & B & A & - & - & A & A & A & A & A & - & A \\
\hline DICO 4 & A & - & - & - & B & - & - & - & $\overline{-}$ & - & $\bar{R}$ \\
\hline DICO 5 & $\mathrm{~B}$ & B & A & B & $\mathrm{B}$ & B & B & B & B & B & B \\
\hline
\end{tabular}

$1 \mathrm{~A}$, above grand mean.

$2 \mathrm{~B}$, below grand mean.

$3-$, at or near grand mean.

giving a detailed account of all characteristics of each DICO cluster as follows (all \% to nearest integer):

\section{DICO Cluster 1}

Size. $\quad \mathrm{N}=32,13 \%$ of the classifiable subjects, $10 \%$ of the total sample.

$A B / F O P . \quad 23$ of the 32 subjects $(72 \%)$ deviated in the minus direction (A pt. anterior to $\mathrm{B} \mathrm{pt}$; relative mandibular retrusion), $6(19 \%)$ deviated in the plus direction (relative mandibular protrusion). No data were obtainable on $3(9 \%)$ of the 32 subjects.

MAX/MAN/BAL. 24 (75\%) MAX, 4 (12\%) MAN, and $4(12 \%)$ BAL.

Prim groups. 23 of the $32(72 \%)$ were also in alpha, $9(28 \%)$ were in beta. None of these subjects appeared in gamma.

Modified angle classification. 5 of the subjects $(15 \%)$ were identified as Class I, $26(81 \%)$ Class II, and $1(3 \%)$ as Class III.

Skeletal characteristics. Mandibular length and ramus alignment tend toward maxillary protrusion; the cranial base does not contribute to the morphology of this group; however, the midface is involved because it is unduly long vertically. The ramus, which usually compensates the maxillary protrusive factors, aggravates the maxillary protrusion in this group.

\section{DICO Cluster 2}

Size. $\quad \mathrm{N}=29,12 \%$ of the classifiable subjects, $9 \%$ of the total sample.

$A B / F O P . \quad 17$ of the 29 subjects $(59 \%)$ deviated in the minus direction (relative mandibular retrusion), $7(24 \%)$ deviated in the plus direction, $3(10 \%)$ were within one standard deviation of the mean. No data were obtainable on $2(7 \%)$ of the 29 subjects.

MAX/MAN/BAL, $22(76 \%)$ MAX, 4 (14\%) MAN, and $3(10 \%)$ BAL.

Prim groups. 20 of the 29 subjects $(69 \%)$ were also in alpha, $9(31 \%)$ were in beta. None of these subjects appeared in gamma. 
Modified angle classification. 17 subjects (59\%) were identified as Class I and $12(41 \%)$ as Class II. None were deemed to be Class III.

Skeletal characteristics. In this group the cranial base is contributory but vertical midface is not (see cluster 1). The ramus acts to compensate for the maxillary protrusive effects of the cranial base and mandibular length, in contrast with its role in DICO cluster 1 .

\section{DICO Cluster 3}

Size. $\quad \mathrm{N}=35,14 \%$ of the classifiable subjects, $11 \%$ of the total sample.

$A B / F O P . \quad 20$ of the 35 subjects $(57 \%)$ deviated in the minus direction (relative mandibular retrusion), 9 (26\%) deviated in the plus direction. No data were obtainable on $6(17 \%)$ of the 35 subjects.

MAX/MAN/BAL. 21 (60\%) $\mathrm{MAX}, 7$ (20\%) MAN, and $7(20 \%)$ BAL.

Prim groups. 27 of the 35 subjects $(77 \%)$ were also in alpha, $8(23 \%)$ were in beta. None of these subjects appeared in gamma.

Angle classification. 13 subjects ( $38 \%$ ) were identified as Class I, $19(54 \%)$ as Class II, and $3(9 \%)$ as Class III.

Skeletal characteristics. The cranial base is tilted upwards anteriorly and, together with the ramus alignment, occlusal plane alignment and mandibular length, is associated with net maxillary protrusion. In this group, the ramus is neither compensatory nor does it aggravate the pattern. The vertical midface is disproportionately long.

\section{DICO Cluster 4}

Size. $\quad \mathrm{N}=99,41 \%$ of the classifiable subjects, $32 \%$ of the total sample.

$A B / F O P . \quad 61$ of the 99 subjects $(62 \%)$ deviated in the plus direction (relative mandibular protrusion), $9(9 \%)$ deviated in the minus direction. No data were obtainable on 29 (29\%) of the subjects.

MAX/MAN/BAL. $13(13 \%)$ MAX, 40 (40\%) MAN, and $46(46 \%)$ BAL.

Prim groups. $20(20 \%)$ of the 99 subjects were also in alpha, $76(77 \%)$ were in beta, and $1(1 \%)$ was in gamma (gamma$1)$. No data were obtainable on $2(2 \%)$ of the subjects.

Modified angle classification. $58(59 \%)$ of the subjects were identified as Class I,
$38(38 \%)$ as Class II, and $3(3 \%)$ as Class III.

Sheletal characteristics. In cluster 4, all the structural counterparts are in balance except the posterior cranial base and mandibular length which normally counterbalance each other.

\section{DICO Cluster 5}

Size. $\quad \mathrm{N}=48,20 \%$ of the classifiable subjects, $16 \%$ of the total sample.

$A B / F O P . \quad 41$ of the 48 subjects $(85 \%)$ deviated in the plus direction (relative mandibular protrusion), 1 (2\%) deviated in the minus direction. No data were obtainable on $6(13 \%)$ of the subjects.

MAX/MAN/BAL. 1 (2\%) MAX, 47 (98\%) MAN, and 0 in the BAL category.

Prim groups. None of these subjects were also in alpha, 23 (48\%) were in beta, and $25(52 \%)$ were in gamma (13 in gamma-1 and 12 in gamma-2). All of the subjects in gamma-2 were also in DICO Cluster 5.

Modified angle classification. Seven $(15 \%)$ of the subjects were identified as Class I, $3(6 \%)$ as Class II, and $38(79 \%)$ as Class III.

Skeletal characteristics. The mandibular arch is relatively longer than the maxillary, when measured from either skeletal or dental landmarks. There is a short vertical midface and the corpus of the mandible is aligned in a downward direction, while the ramus is aligned in a forward direction.

The salient features are summarized in table 4 , which gives, for each DICO cluster, the largest percentage group within AB/FOP, MAX/MAN/BAL, Prim groups, and Angle classification.

\section{Cluster analysis of variables}

These results are presented in table 5 . The reliability coefficient gives a measure of the degree in which the behavior of any one variable in a cluster resembles the behavior of any other variable. If the reliability coefficient of a cluster is high enough (a subjective evaluation), then one variable may be chosen as a representative of the entire cluster.

For example, in the first variable cluster, either variable 1 or variable 2 may be selected as representative of both. In the 
TABLE 4

Summary of characteristics of DICO clusters

\begin{tabular}{|c|c|c|c|c|}
\hline $\begin{array}{l}\text { DICO } \\
\text { cluster }\end{array}$ & $\mathrm{AB} / \mathrm{FOP}$ & MAX/MAN/BAL & Prim group & $\begin{array}{c}\text { Angle } \\
\text { classification }\end{array}$ \\
\hline 1 & $\begin{array}{c}72 \% \\
\text { A pt. anterior } \\
\text { to } \mathrm{B} \text { point }\end{array}$ & $\begin{array}{l}75 \% \\
\text { MAX }\end{array}$ & $\begin{array}{l}72 \% \\
\text { alpha }\end{array}$ & $\begin{array}{l}69 \% \\
\text { II }\end{array}$ \\
\hline 2 & $\begin{array}{c}59 \% \\
\text { A pt. anterior } \\
\text { to B point }\end{array}$ & $\begin{array}{l}76 \% \\
\text { MAX }\end{array}$ & $\begin{array}{l}69 \% \\
\text { alpha }\end{array}$ & $\begin{array}{c}45 \% \\
\text { I } \\
\ldots \ldots \\
34 \% \\
\text { II }\end{array}$ \\
\hline 3 & $\begin{array}{l}57 \% \\
\text { A pt. anterior } \\
\text { to B point }\end{array}$ & $\begin{array}{l}60 \% \\
\text { MAX }\end{array}$ & $\begin{array}{l}77 \% \\
\text { alpha }\end{array}$ & $\begin{array}{c}51 \% \\
\text { II }\end{array}$ \\
\hline 4 & $\begin{array}{c}62 \% \\
\text { B pt. anterior } \\
\text { to A point }\end{array}$ & $\begin{array}{l}46 \% \\
\text { BAL } \\
\dddot{2} \% \\
\text { MAN }\end{array}$ & $\begin{array}{l}77 \% \\
\text { beta }\end{array}$ & $\begin{array}{c}55 \% \\
I\end{array}$ \\
\hline 5 & $\begin{array}{c}85 \% \\
\text { B pt. anterior } \\
\text { to A point }\end{array}$ & $\begin{array}{l}98 \% \\
\text { MAN }\end{array}$ & $\begin{array}{c}52 \% \\
\text { gamma } \\
\ldots \ldots \\
48 \% \\
\text { beta }\end{array}$ & $\begin{array}{l}79 \% \\
\text { III }\end{array}$ \\
\hline
\end{tabular}

second cluster one may select either variable 7 or variable 9 and so on.

However, variables 3,5 and 6 do not appear in any cluster. Therefore, they are unique (not collinear with any of the others), and must be evaluated separately. That is, any reduced list of the original 13 variables must contain them.

The reduced list obtained from table 5 consists of variables $3,4,5,6,7,8$ and 13 . Alternatively, variable 1 may be used for an overall evaluation of the facial profile. (Remember that variables 1 and 2 are aggregates or summaries of the other 11 variables.) The choices made within any one cluster depended on ease and accuracy of measurement.

\section{Analysis of ambiguously classified subjects}

The term "ambiguous" refers to subjects that were equidistant from two or more DICO centers, and so could not be grouped unequivocally with any one center.

There were 65 such subjects $(21.1 \%$ of the total sample). Three of these were discarded because of inadequate data, leaving 62 for further study. The analysis
TABLE 5

Clusters of collinear variables

\begin{tabular}{ccc}
\hline $\begin{array}{c}\text { Cluster } \\
\text { no. }\end{array}$ & $\begin{array}{c}\text { Variables } \\
\text { in cluster }\end{array}$ & $\begin{array}{c}\text { Reliability } \\
\text { coefficient }\end{array}$ \\
\hline 1 & 1,2 & 0.9242 \\
2 & 7,9 & 0.9358 \\
3 & 4,11 & 0.8417 \\
4 & 8,10 & 0.8422 \\
5 & 12,13 & 0.7003 \\
\hline
\end{tabular}

of this group, taken as a whole, with regard to $\mathrm{AB} / \mathrm{FOP}, \mathrm{MAX} / \mathrm{MAN} / \mathrm{BAL}$, and Angle classification is:

AB/FOP. 24 of the 62 subjects $(39 \%)$ deviate in the minus direction, $28(45 \%)$ deviate in the plus direction, $2(3 \%)$ were within one standard deviation from the mean. No data were obtainable on $8(13 \%)$ of these subjects.

MAX/MAN/BAL. 32 (52\%) MAX, 15 (24\%) MAN, $15(24 \%)$ BAL.

Prim groups. 39 of the $62(63 \%)$ were in alpha, $23(37 \%)$ in beta. None of these subjects was in gamma.

Modified angle classification. $28(45 \%)$ of the subjects were identified as Class I, $29(47 \%)$ as Class II, and $5(8 \%)$ as Class III. 
Upon analysis of the groups separately for $\mathrm{AB} / \mathrm{FOP}, \mathrm{MAX} / \mathrm{MAN} / \mathrm{BAL}$, and Angle classification, the following results were obtained (summarized in table 6).

(1) Subjects grouped with 2 or more of DICO 1, 2, and 3.

Size. $\quad \mathrm{N}=19(31 \%$ of the ambiguous subjects).

$A B / F O P .13$ of the 19 subjects $(68 \%)$ deviated in the minus direction, $5(26 \%)$ deviated in the plus direction. No data were obtainable for $1(5 \%)$ of the subjects.

$M A X / M A N / B A L . \quad 17$ (89\%) MAX, 0 $(0 \%)$ MAN, and $2(11 \%)$ BAL.

Prim groups. 16 of the $19(84 \%)$ were in alpha, $3(16 \%)$ in beta. None of these subjects appeared in gamma.

Modified angle classification. Eight of the subjects $(42 \%)$ were identified as Class I, $10(53 \%)$ as Class II and $1(5 \%)$ as Class III,

(2) Subjects grouped with 1 or more of DICO 1, 2, and 3, and with DICO 4.

Size. $\mathrm{N}=35$.

$A B / F O P . \quad 11$ of the 35 subjects $(31 \%)$ deviated in the minus direction, $18(51 \%)$ deviated in the plus direction, and $1(3 \%)$ was within 1 standard deviation of the mean. No data were obtainable for 5 (14\%) of the 35 subjects.

MAX/MAN/BAL. 14 (40\%) MAX, 11 (31\%) MAN, and $10(29 \%)$ BAL.

Prim groups. $21(60 \%)$ were in alpha, $14(40 \%)$ were in beta. None of these subjects appeared in gamma.

Modified angle classification. 15 of the subjects $(43 \%)$ were identified as Class I, $18(51 \%)$ as Class II, and $2(6 \%)$ as Class III. Of the 18 Class II subjects, 10 deviated in the minus direction, and also were classified as MAX, thus accounting for most of the incidence of minus and MAX reported above for this ambiguous group.

(3) Subjects grouped with 1 or more of DICO 1, 2, 3, and 4, and with DICO 5.

Size. $\mathbf{N}=8$.

$A B / F O P$, None of the 8 deviated in the minus direction, $5(62 \%)$ deviated in the plus direction, and $1(12 \%)$ was within 1 standard deviation of the mean. No data were obtainable on $2(25 \%)$ of the subjects.

MAX/MAN/BAL. 1 (12\%) MAX, 5 (62\%) MAN, $2(25 \%)$ BAL.

Prim groups. All $8(100 \%)$ of the subjects were in beta.

Modified angle classification. Five of the subjects $(62 \%)$ were identified as Class I, $1(13 \%)$ as Class II and $2(25 \%)$ as Class III.

\section{DISCUSSION}

With regard to the questions set in the introduction, the results clearly show that it is possible to derive, by numerical methods of taxonomy, meaningful categories of human skeletal facial types.

The most important feature of the numerical taxonomic approach is that the groupings are derived completely objectively by the clustering techniques employed. These operate directly on the data as a whole to find natural relationships that may exist among the subjects. No prearrangement of the subjects is done in any way by the investigator. Therefore, the clusters found do not represent any prejudice or externally imposed classification.

The important aspect of our application of the numerical approach is that the

TABLE 6

Summary of characteristics of ambiguously classified subjects

\begin{tabular}{|c|c|c|c|c|c|}
\hline & Subjects grouped with & $A B / F O P$ & MAX/MAN/BAL & Prim groups & $\begin{array}{c}\text { Modified } \\
\text { angle class }\end{array}$ \\
\hline (1) & $\begin{array}{l}2 \text { or more of DICO } 1, \\
2, \text { and } 3\end{array}$ & $\begin{array}{l}68 \% \\
\text { A pt. anterior } \\
\text { to B point }\end{array}$ & $\begin{array}{l}89 \% \text { MAX } \\
11 \% \text { BAL }\end{array}$ & $\begin{array}{l}84 \% \text { alpha } \\
16 \% \text { beta }\end{array}$ & $\begin{array}{l}42 \% \text { I } \\
58 \% \text { II }\end{array}$ \\
\hline (2) & $\begin{array}{l}1 \text { or more of DICO } 1 \text {, } \\
2, \text { and } 3, \text { and with } \\
\text { DICO } 4\end{array}$ & $\begin{array}{l}51 \% \\
\text { B pt. anterior } \\
\text { to A point }\end{array}$ & $\begin{array}{ll}40 \% & \text { MAX } \\
31 \% & \mathrm{MAN} \\
29 \% & \mathrm{BAL}\end{array}$ & $\begin{array}{l}60 \% \text { alpha } \\
40 \% \text { beta }\end{array}$ & $\begin{aligned} 43 \% & \text { I } \\
51 \% & \text { II } \\
6 \% & \text { III }\end{aligned}$ \\
\hline (3) & $\begin{array}{l}1 \text { or more of DICO } 1,2 \text {, } \\
3 \text {, and } 4 \text {, and with } \\
\text { DICO } 5\end{array}$ & $\begin{array}{l}62 \% \\
\text { B pt. anterior } \\
\text { to A point }\end{array}$ & $\begin{array}{ll}12 \% & \text { MAX } \\
62 \% & \text { MAN } \\
25 \% & \text { BAL }\end{array}$ & $100 \%$ beta & $\begin{array}{ll}63 \% & \text { I } \\
13 \% & \text { II } \\
25 \% & \text { III }\end{array}$ \\
\hline
\end{tabular}


existence of the derived categories is based not on one single analytic method, but on the cumulative evidence obtained from two different techniques of cluster analysis (Prim and DICO), a separate overall evaluation (MAX/MAN/BAL), a traditional classification (Angle), and an independent measure of maxillo-mandibular relation$\operatorname{ship~(AB/FOP).~}$

Five categories of profiles have been derived in this way. They represent a composite of the Prim and DICO designations, and henceforth will be called Category A, Category B, etc. Categories A-D correspond to DICO 1-4, and Category E contains within it the subgroups gamma-1 and gamma-2 detected in the Prim analysis. We suspect that one of these subgroups represents subjects in which the imbalances consist of absolute mandibular protrusion (maxilla in normal range) and that the other represents subjects in which the imbalances are compounded of maxillary retrusion together with mandibular protrusion. The latter might be expected to be more severe, the former less severe.

Table 7 provides, for easy reference, a list summarizing the distinguishing characteristics of each of the five categories along with the major associated morphological features, expressed in the terminology devised by Enlow (Enlow and Moyers, '71; Enlow et al., '71a,b). Category D, referred to as balanced profiles, actually represents a spectrum ranging from near maxillary protrusion to near mandibular protrusion, and warrants further study in itself.

The existence of distinguishing characteristics among the five categories makes possible the design of an identification procedure that would allow one to place a given, new individual reliably into one of the categories. This represents a separate problem not included in these studies. However, the framework of such a procedure has been revealed in the present work and is presented in figures 2 and 3.

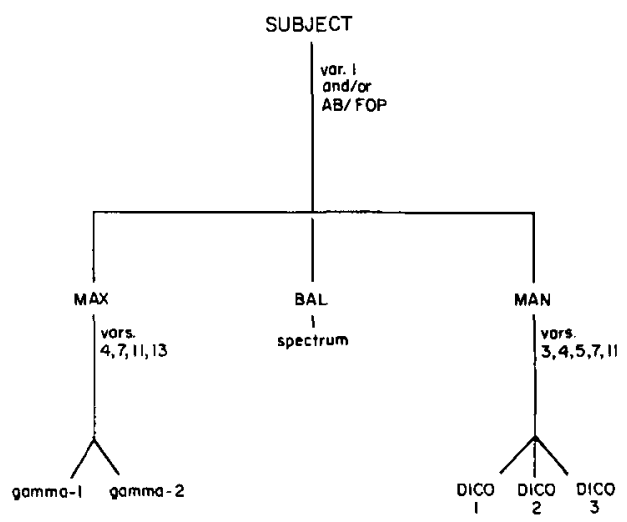

Fig. 2 Basis for a classification scheme. The variable numbers refer to the list given in table 1 .

TABLE 7

Characteristics and associated morphological features of the derived categories

\begin{tabular}{ccc}
\hline Category & Characteristic & $\begin{array}{c}\text { Major associated } \\
\text { morphological features }\end{array}$ \\
\hline A & Maxillary protrusion & $\begin{array}{c}\text { Maxillary arch - mandibular } \\
\text { corpus imbalance, long vertical } \\
\text { midface, noncompensatory align- } \\
\text { ment of mandible (ramus) }\end{array}$ \\
B & Maxillary protrusion & $\begin{array}{c}\text { Maxillary arch - mandibular } \\
\text { corpus imbalance, cranial } \\
\text { base flexure }\end{array}$ \\
C & Maxillary protrusion & $\begin{array}{c}\text { Cranial base flexure, long } \\
\text { vertical midface }\end{array}$ \\
& $\begin{array}{c}\text { Balanced profile in a } \\
\text { spectrum from maxil- } \\
\text { lary to mandibular } \\
\text { protrusion }\end{array}$ & $\begin{array}{c}\text { Spectrum caused mainly by } \\
\text { degree of posterior cranial } \\
\text { base - ramus imbalance }\end{array}$ \\
& $\begin{array}{c}\text { Mandibular protrusion } \\
\text { moderate to severe }\end{array}$ & $\begin{array}{c}\text { Long mandibular arch, short } \\
\text { vertical midface, protrusive } \\
\text { alignment of the corpus of } \\
\text { the mandible }\end{array}$ \\
\hline
\end{tabular}


Figure 2 is in terms of the temporary designations used in the cluster analysis. It shows that the use of variable 1 (see list in table 1) and/or $\mathrm{AB} / \mathrm{FOP}$ will serve to place an individual into one of the three major profile groupings. Further classification within the MAX and MAN groups requires the information supplied by the variables indicated.

Figure 3 is essentially the same classification scheme except that the temporary designations have been replaced by the category names, and the variable numbers have been replaced by the major structural features characteristic of each category.

Figure 3, therefore, provides a first step towards a classification key such as is used by biologists in identifying unknown specimens. Inspection of table 5 will reveal that the reduced lists of variables obtained from the cluster analysis of variables are used as the key variables appearing in the classification scheme. These are the variables that would convey, collectively, an acceptably large percentage and broad range of information about a subject under study.
Through the use of these variables in the manner indicated in figure 3 , one could expect to be able to place nearly $80 \%$ of subjects unambiguously into one of the five categories; the remaining $20 \%$ would overlap two or more categories (actual figures $78.9 \%$ and $21.1 \%$, table 2). The establishment of the expectation of an $80 \%$ chance of accurate identification, together with the description of the five provisional categories satisfy the major aims of this work.

With regard to the correspondence of the traditional Angle classes and the categories derived by the numerical approach, Categories $\mathrm{A}, \mathrm{B}$ and $\mathrm{C}$ may be regarded as subgroups, heretofore unrevealed, of Angle Class II. Category E contains evidence of two subgroups within Angle Class III. Category D is an unresolved spectrum as is Angle Class I.

We believe that further resolution of the groupings within the categories, especially Category D and the further resolution of the ambiguous subjects are related problems. The same considerations of measurement technique and uniformity of sample (the present sample is not uniform

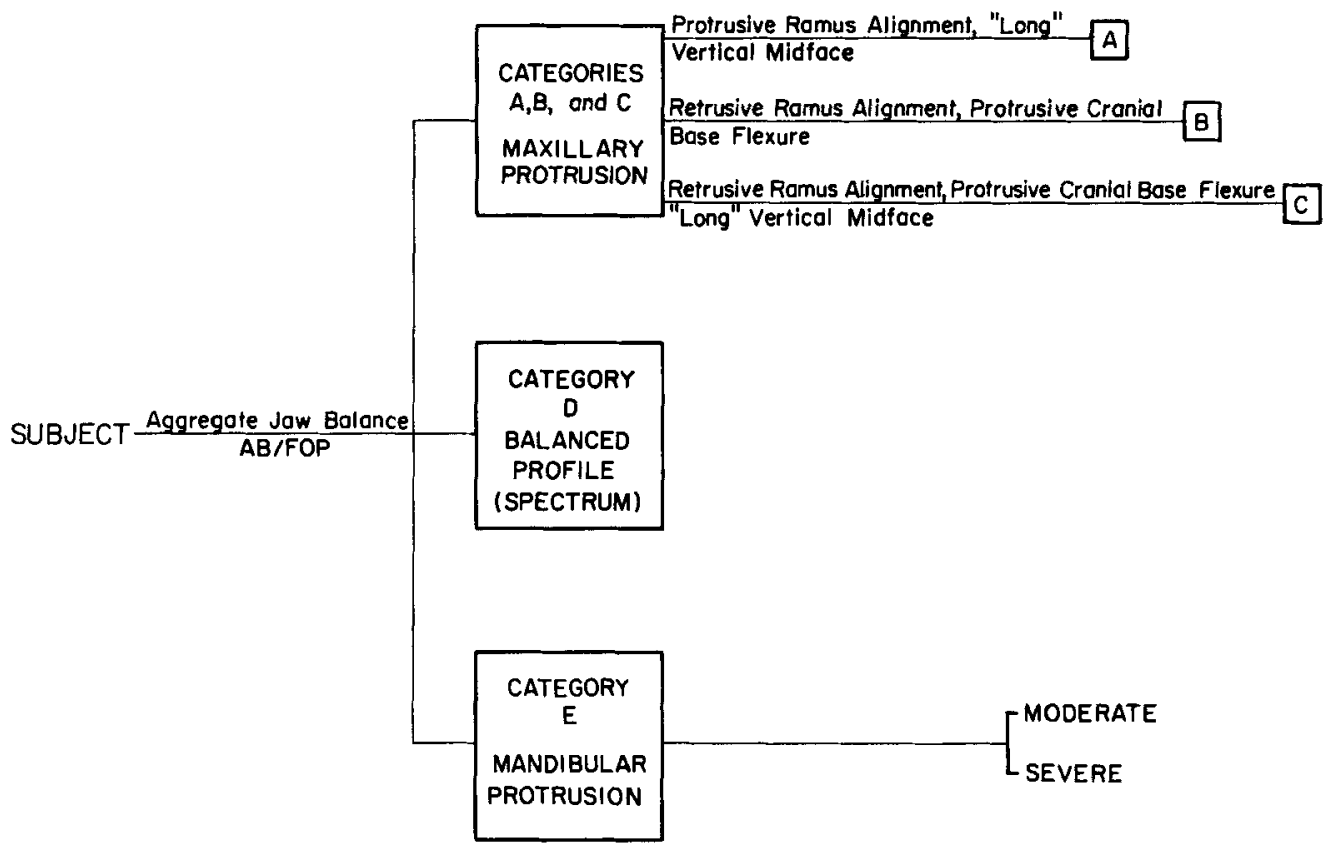

Fig. 3 Suggested form of a taxonomic key of skeletal facial types. The types include only those derived from lateral cephalograms as reported in this work. 
with respect to age and sex) apply to both problems. Some of the fuzziness of the groupings may have linguistic grounds.

However, the intent of this paper is to disseminate to workers in other specialties knowledge of an approach that has been of great heuristic value to us in our own primary specialties. A deeper exposition of skeletal typing with regard to craniofacial biology and orthodontics would carry us too far afield.

Our message is that the combined numerical and subjective approach may be used profitably by anyone faced with the problem of defining and labeling subgroups of a population.

\section{ACKNOWLEDGMENTS}

This research was supported in part by grant NIH-HD-2272, National Institute of Child Health and Human Development. Technical assistance was provided by Anthony Gaston and Ruth Bigio.

\section{LITERATURE CITED}

Angle, E. H. 1970 Treatment of Malocclusion of the Teeth and Fractures of the Maxillae. Seventh ed. S. S. White Dental Mfg. Co., Philadelphia.
Blacklith, R. E., and R. A. Reyment 1971 Multivariate Morphometrics. Academic Press, New York, pp. 338-343.

Brown, T., M. J. Barrett and J. N. Darroch 1965 Craniofacial factors in two ethnic groups. Growth, 29: 109-123.

Enlow, D. H., and R. E. Moyers 1971 Growth and architecture of the face. J. Am. Dent. Assoc., 82: 763-774.

Enlow, D. H., T. Kuroda and A. B. Lewis 1971a The morphological and morphogenetic basis for craniofacial form and pattern. Angle Orthodont., 41: 161-188.

- 1971b Intrinsic craniofacial compensations. Angle Orthodont., 41: 271-285.

Farris, J. S. 1970 Methods for computing Wagner trees. Syst. Zool., 19: 83-92.

Gower, J. C. 1967 A comparison of some methods of cluster analysis. Biometrics, 23: 623-637.

Howells, W. W. 1969 The use of multivariate techniques in the study of skeletal populations. Am. J. Phys. Anthrop., 31: 311-314.

1971 Applications of multivariate analysis to craniofacial growth in man. In: Craniofacial Growth in Man. R. E. Moyers and W. Krogman, eds. Pergamon Press, pp. 209-218.

Prim, R. C. 1957 Shortest connection networks and some generalizations. Bell Syst. Tech. J., 36: 1389-1401.

Rohlf, F. J. 1970 Adaptive hierarchial clustering schemes. System Zool., 19: 58-82.

Sokal, R. R., and P. A. H. Sneath 1963 Principles of Numerical Taxonomy. W. H. Freeman and Co., San Francisco.

Tryon, R. C., and D. E. Bailey 1971 Cluster Analysis. McGraw-Hill, New York. 